Research Paper

\title{
Correlation of GOLPH3 Gene with Wnt Signaling Pathway in Human Colon Cancer Cells
}

\author{
Cheng-Zhi Qiu ${ }^{\bowtie}$, Ming-Zhen Wang, Wai-Shi Yu, Yan-Ta Guo, Chun-Xiao Wang, Xiao-Feng Yang \\ Department of General Surgery, the Second Affiliated Hospital of Fujian Medical University, Quanzhou 362000, Fujian Province, China. \\ $\triangle$ Corresponding author: Cheng-Zhi Qiu, Department of General Surgery, the Second Affiliated Hospital of Fujian Medical University, Quanzhou 362000, \\ Fujian Province, China. qchengzhi@sohu.com. \\ (C) Ivyspring International Publisher. Reproduction is permitted for personal, noncommercial use, provided that the article is in whole, unmodified, and properly cited. See \\ http://ivyspring.com/terms for terms and conditions.
}

Received: 2015.09.26; Accepted: 2016.03.16; Published: 2016.05.07

\begin{abstract}
Objective: Overexpression of GOLPH3 in colorectal cancer tissue may promote cell proliferation and activate the Wnt signaling pathway. We investigated the correlation between GOLPH3 gene expression and the Wnt signaling pathway to explore the mechanism of the overexpression of GOLPH3 gene which promotes proliferation in human colon cancer cells.

Methods: We measured expression of GOLPH3 mRNA in the human colon cancer cell lines HCT116, HT29, SW480 and SW620 by RT-PCR, and the cells with the highest expression were selected and divided into four groups: negative control, GOLPH3 siRNA transfection (siRNA-GOLPH3), Akt inhibitor (Tricinbine), and glycogen synthase kinase (GSK)-3 $\beta$ inhibitor (TWS1 19). After human colon cancer cells were transfected with siRNA-GOLPH3, we used RT-PCR to investigate the silencing effect of GOLPH3 gene. We assessed the activity of the Wnt signaling pathway in all groups using the Topflash method. Proliferation and apoptosis of colon cancer SW620 cells were detected by MTT assay, colony formation assay and flow cytometry. Expression of Golgi phosphoprotein (GOLPH)3, $\beta$-catenin, GSK-3 $\beta$ and $p S 9-G S K-3 \beta$ in cancer cells was determined by Western blotting.

Results: SW620 cells expressed the highest level of GOLPH3 mRNA, and the silence effect was good after they were transfected with siRNA-GOLPH3. The relative luminescence units (RLU) values in the experimental groups were significantly lower than in the negative control group $(P<0.001)$. There was no significant difference in the RLU values among the experimental groups $(P>0.05)$. The growth inhibition ratio and apoptosis rate of cancer cells in each experimental group were significantly higher than those in the control group, and the cell colony count in the experimental group was significantly lower than in the control group $(P<0.05)$. In addition, the RLU value, proliferation and apoptosis rate of cancer cells did not differ significantly between each two experimental groups. Western blotting showed that, compared with the control group, expression of $\beta$-catenin and pS9-GSK 3 proteins were significantly decreased in the experimental group. Expression of GSK-3 $\beta$ in the experimental group did not different from that of the control group.

Conclusions: Overexpression of GOLPH3 gene activated the Wnt signaling pathway, as well as increasing expression of $\beta$-catenin, promoting proliferation and inhibiting apoptosis in human colon cancer cells. The mechanism of action was that overexpression of GOLPH3 gene activated Akt, which may also further activate the Wnt signaling pathway via GSK-3 $\beta$, and promote proliferation in human colon cancer cells.
\end{abstract}

Key words: Colon cancer cells; GOLPH3; Wnt signaling pathway; Akt; glycogen synthase kinase-3 $\beta$; $\beta$-catenin.

\section{Introduction}

Colon cancer is the most common of the digestive neoplasms and the fourth most frequent cause of cancer-related death worldwide, and its incidence continues to show an upward trend.
Carcinogenesis and progression of colon cancer are still unclear, but the disease course is accompanied by numerous genetic alterations, including oncogenes and tumor suppressor genes, such as P53, PTEN and 
K-ras. These genes act on different loci in the intracellular signal transduction cascade, and influence cancer cell growth, proliferation and apoptosis. There are two important intracellular signaling pathways in colon cancer, $\mathrm{PI} 3 \mathrm{~K} / \mathrm{Akt} / \mathrm{mTOR}$ and Wnt/ $\beta$-catenin, which are closely related to differentiation, proliferation, migration and adhesion of cancer cells, and also have many attractive potential targets ${ }^{1}$.

Golgi phosphoprotein (GOLPH)3, also known as GPP34, is localized on human chromosome 5p13; a region that is frequently amplified in several types of solid tumor. Recent studies indicate that GOLPH3 can activate the $\mathrm{PI} 3 \mathrm{~K} / \mathrm{AKT} / \mathrm{mTOR}$ signaling pathway through phosphorylation both of mammalian target of rapamycin complex (mTORC) 1 and mTORC2, resulting in cell proliferation ${ }^{2-4}$. GLOPH3 is a novel oncogene that is overexpressed in prostate cancer ${ }^{5}$, gastric cancer ${ }^{6,7}$, hepatic carcinoma ${ }^{8,9}$ and pancreatic ductal adenocarcinoma ${ }^{10}$, but also correlated with poor prognosis.

Besides the PI3K/Akt/mTOR signaling pathway, the other mechanism by which GOLPH3 promotes cell proliferation remains uncertain. Our previous study proved that overexpression of GOLPH3 was significantly correlated with $\beta$-catenin expression in colorectal cancer tissue. We propose that GOLPH3 can activate the intracellular Wnt/ $\beta$-catenin signaling pathway and promote cell proliferation. The objectives of this study were to investigate the relationship between GOLPH3 overexpression and activity of the Wnt signaling pathway in colon cancer cells, and further explore its possible mechanism via inhibition of Akt or glycogen synthase kinase (GSK)-3 $\beta$. We found that knockdown of GOLPH3 inhibited human colon cell proliferation, $\beta$-catenin expression and activity of the Wnt signaling pathway, and induced apoptosis. The mechanism included GOLPH3 gene activation of Akt and enhancement of Wnt signaling mediated by GSK-3 $\beta$, which resulted in proliferation of human colon cancer cells.

\section{Materials and Methods}

\section{Cell lines and cell culture}

Four human colorectal cancer cell lines HCT116, HT129, SW480 and SW620 were purchased from the Type Culture Collection of the Chinese Academy of Sciences (Shanghai, China). All cell lines were maintained in RPMI-1640 medium (Invitrogen, Carlsbad, CA, USA) supplemented with $10 \%$ fetal bovine serum and grown in a humidified incubator with $5 \% \mathrm{CO}_{2}$ at $37^{\circ} \mathrm{C}$.

\section{RNA extraction and quantitative RT-PCR}

Total RNA was extracted from cultured cells using the TRIzol reagent (Invitrogen). Two micrograms of total RNA from each sample was reverse transcribed into cDNA using SuperScript III Reverse Transcriptase kit (Invitrogen). Quantitative PCR was performed using the SYBR Ex Taq kit (Takara Bio, Japan) and the ABI 9700 qRT-PCR detection system (Applied Biosystems, Foster City, CA, USA). The quantitative PCR conditions were initial degeneration for $20 \mathrm{~s}$ at $95^{\circ} \mathrm{C}$, followed by 45 cycles of denaturation for $10 \mathrm{~s}$ at $95^{\circ} \mathrm{C}$, annealing for $20 \mathrm{~s}$ at $60^{\circ} \mathrm{C}$ and extension for $20 \mathrm{~s}$ at $72^{\circ} \mathrm{C}$. All PCR primers were synthesized by Zimmer AG (Shanghai, China). The primers sequences were as follows. GOLPH3 forward: 5'-AGGGCGACTCCAAGGAAA3'; reverse: 5'-TGATGTGTAACCCTCGCG-3' and primers to amplify GAPDH contained: 5'-GGTCATAAGCTTGCGTTGATTAAG-3' (forward) and 5'-CTACGGAAACCTTGTTACGACTTT-3' (reverse). Using GAPDH as an internal reference, the relative expression level was calculated by using the $2^{-\triangle \Delta C T}$ equation.

Colon cancer cell lines with high expression of GOLPH3 were divided into four groups: control group (Equivalent PBS), siRNA transfection group, Tricinbine group (Akt inhibitor) and TWS119 group (GSK-3 $\beta$ inhibitor). We continued to culture the cells, and detected expression of GOLPH3 mRNA in the group with siRNA transfection, as for the RT-PCR method.

\section{Determination of Wnt signaling pathway activity}

The culture medium was removed and the cells washed with PBS. When adding $100 \mu \mathrm{l}$ Harvest buffer to lyse the cells 10 min later, we withdrew $40 \mu$ lysate into the centrifuge tube and added $20 \mu$ lirefly luciferase substrate or Renilla luciferase substrate (Beyotime Biotechnology, Nanking, China). Once mixing evenly by vortex, we detected firefly and Renilla luciferase fluorescence with a photometer (Beckman Coulter America). The ratio of the above two values [relative luminescence units (RLU) value] indicated the activity of cellular transcription factors in the Wnt signaling pathway.

\section{Cell proliferation (MTT) assay}

The cell suspension was inoculated into a 96-well plate, with each well receiving $100 \mu \mathrm{l}\left(10^{5}\right.$ cells), and cultured by the anchorage-dependent way for $24 \mathrm{~h}$. Next each group set up four double wells and four control wells that only contained cells without any other treatment. After $48 \mathrm{~h}$ culture, $10 \mu \mathrm{l}$ of $5 \mathrm{mg} / \mathrm{ml}$ MTT (Sigma, St Louis, MO, USA) was added to each well, followed by further culture for $4 \mathrm{~h}$, after which, the culture medium was discarded. To each well, we 
added $150 \mu \mathrm{l}$ DMSO (Sigma), and the wells were mixed by oscillation in the dark. $\mathrm{OD}_{490}$ was measured with a microplate reader (Huadong Electronics, China) after the crystals dissolved. The formula: Rate of growth inhibition in cancer cells $=(1-$ average $\mathrm{OD}_{490}$ in treatment groups/average $\mathrm{OD}_{490}$ in control groups) $\times 100 \%$.

\section{Colony formation assay}

Cells in logarithmic growth phase were inoculated into six-well plates containing complete culture medium and 500 cells per well. During the next 3 weeks, the medium was changed once every 3 days. Once the clones became visible, culture was terminated; the cells were washed with PBS and fixed in $100 \%$ methanol. After staining with crystal violet dye for $30 \mathrm{~min}$, the cells were washed three times with PBS and clones were counted under an inverted microscope. Clones were colonies of $\geq 50$ cells. The above steps were repeated three times and the average was calculated.

\section{Apoptotic assay}

Centrifuge the cell suspension at 1,000 rpm for 3 minutes and remove supernatant. Add PBS for wash cells and centrifuge at 1,000 rpm for 3 minutes, remove supernatant. Repeat this step one more time. Add 10-fold diluted Annexin V Binding Solution to make final cell concentration of $1 \times 10^{6}$ cells $/ \mathrm{ml}$. Transfer $100 \mu \mathrm{l}$ of cell suspension to a new tube. Add 5 $\mu l$ of Annexin V, FITC Conjugate, then $5 \mu$ of PI Solution to the cell suspension. Incubate 15 minutes at room temperature with protect from light. Add $400 \mu \mathrm{l}$ of 10-fold diluted Annexin V Binding Solution. Apply the solution to flow cytometric assay (Beckman Coulter, America).

\section{Western blotting analysis}

Each cell line in logarithmic growth phase was washed with PBS. The cells were lysed in RIPA lysis buffer (containing $50 \mathrm{mM}$ Tris- $\mathrm{HCl}, \mathrm{pH} 7.4,150 \mathrm{mM}$ $\mathrm{NaCl}, 0.1 \%$ SDS, $1 \mathrm{mM}$ EDTA, $1 \%$ Triton X-100, $1 \mathrm{mM}$ PMSF, and $1 \mathrm{mM}$ Protease Inhibitor Cocktail) for 20 min on ice with occasional vortex mixing. Protein concentrations were determined using the BCA assay kit (wegenebio, China). Protein concentrates of cell lysates (30 $\mathrm{\mu g}$ per lane) were separated by $10 \%$ SDS-PAGE and transferred electrophoretically to nitrocellulose membranes (solarbio, China). Membranes were blocked with 5\% skimmed milk in TBST for $1 \mathrm{~h}$ at room temperature. The blots were incubated with antibodies against GOLPH3 (Abcam, Cambridge, UK); pS9-GSK-3 $\beta$ (Cell Signaling Technology, Danvers, MA, USA); GSK-3 $\beta$ (Santa Cruz Biotechnology, Santa Cruz, CA, USA); $\beta$-catenin (Santa Cruz Biotechnology); $\beta$-actin (Santa Cruz
Biotechnology) at $4^{\circ} \mathrm{C}$ overnight. After being washed three times with PBST for $10 \mathrm{~min}$, the membranes were incubated with horseradish peroxidaseconjugated secondary antibodies (Santa Cruz Biotechnology) for $1 \mathrm{~h}$ at room temperature. The bands were visualized with a chemiluminescence detection kit (ECL, Rockford, IL, USA) and the result was analyzed by Image J Software. Relative intensity target protein $=$ target band of gray value $/ \beta$-actin band gradation value. $\beta$-Actin was used as a loading control.

\section{Statistical analysis}

SPSS version 19.0 was used for data processing, and data were expressed as mean $\pm \mathrm{SD}$. Dunnett's $t$ test was used to compare the experimental and control groups. Differences between the experimental groups were compared using one-way ANOVA. Differences between paired experimental groups were compared using Student-Newman-Keuls q test. $P<0.05$ indicated significant differences.

\section{Results}

\section{Expression of GOLPH3 in human colon cancer cell lines}

We measured the expression level of GOLPH3 mRNA in colon cancer cell lines HCT116, HT29, SW480 and SW620, using RT-PCR. SW620 cells had the highest level of expression (Figure 1). Compared to the control group, the relative expression of GOLPH3 mRNA in siRNA-transfected group was significantly decreased $(P<0.001)$. The expression level of mRNA GOLPH3 in SW620 carcinoma cells was successfully silenced by siRNA transfection (Figure 2).

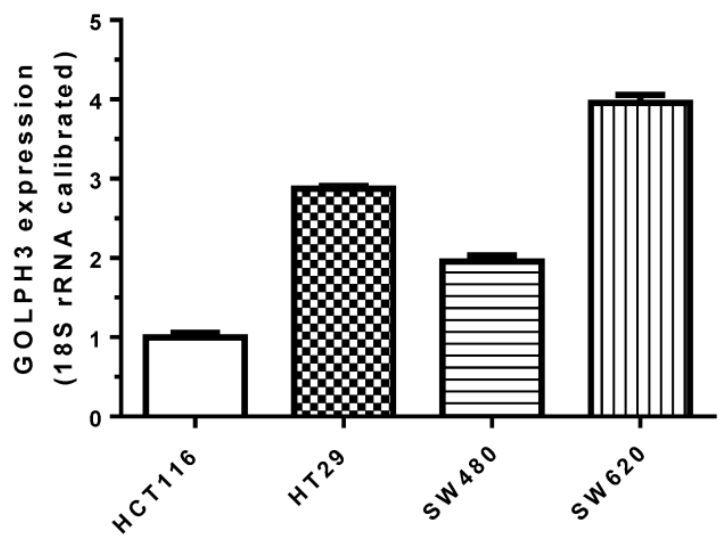

Figure 1. Expression of GOLPH3 mRNA in various colon cancer cell groups. The expression of mRNA GOLPH3 was detected by RT-PCR after four kinds of cancer cells were cultured under the same conditions. 


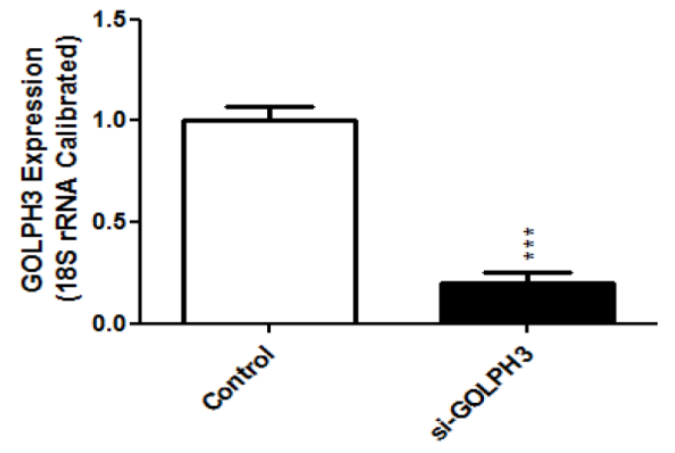

Figure 2. GOLPH3 mRNA expression in control group and transfection group. Values are expressed as percentage of untreated control. *** indicates statistical significance. $P<0.001$ compared with control group.

\section{Detection of Wnt signaling pathway activity}

We used the Topflash reporter assay to compare activation of the Wnt signaling pathway among the cell lines. The RLU values (relative luciferase activity) of the three experimental groups (siRNA-GOLPH3, Tricinbine and TWS119) were significantly lower than in the control group $(P<0.01)$. Compared with each two of the experimental groups, the RLU value did not differ significantly in cancer cells $(\mathrm{P}>0.05)$. Consequently, silencing GOLPH3 gene expression, blocking Akt loci and inhibiting GSK-3 $\beta$ can decrease activation of the Wnt signaling pathway in SW620 colon cancer cells (Figure 3).

\section{Proliferation and apoptosis of cancer cells}

MTT assay showed that each $\mathrm{OD}_{490}$ value of the experimental groups (siRNA-GOLPH3 transfection, Tricinbine and TWS119) was lower than in the control group $(P<0.001)$ (Figure 4). Similar results were found in the plate clone-forming assay (Figure 5), in which the colony number of tumor cells in each experimental group was significantly lower than in the control group $(P<0.001)$. Besides, the apoptosis rate of cancer cells in the experimental group was significantly increased $(P<0.001)$ (Figure 6A, B). These findings revealed that silencing GOLPH3 gene expression, blocking Akt loci and inhibiting GSK-3 $\beta$ all inhibit SW620 colon cancer cell proliferation and promote apoptosis of cancer cells. In addition, the RLU value, proliferation and apoptosis rate of cancer cells did not differ significantly in each two of the experimental groups.

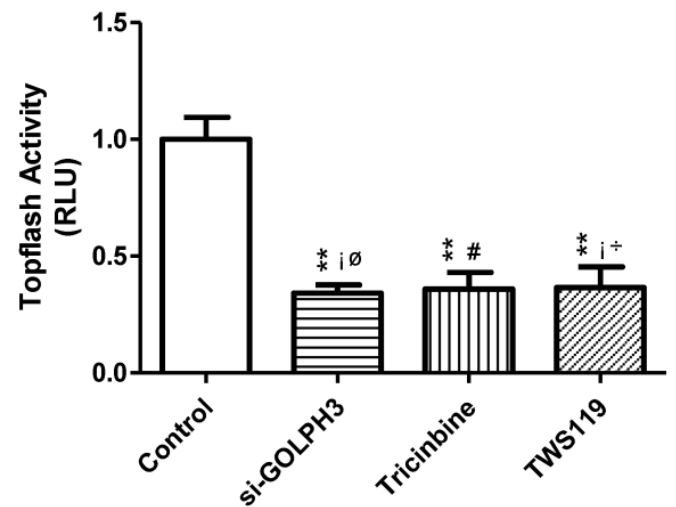

Figure 3. RLU values in various groups of SW620 colon cancer cells. The activity of Wnt signaling pathway in cancer cells was detected by Topflash. Dunnett-t test was used to compare the experimental group and control group, and SNK-q test was used to compare the paired experimental groups. **: $P<0.01$ compared with control group; $\boldsymbol{\Delta}: P>0.05$ compared with Tricinbine group; \#: $P>0.05$ compared with TWS1 19 group; $\triangle: P>0.05$ compared with si-Golph3 group.

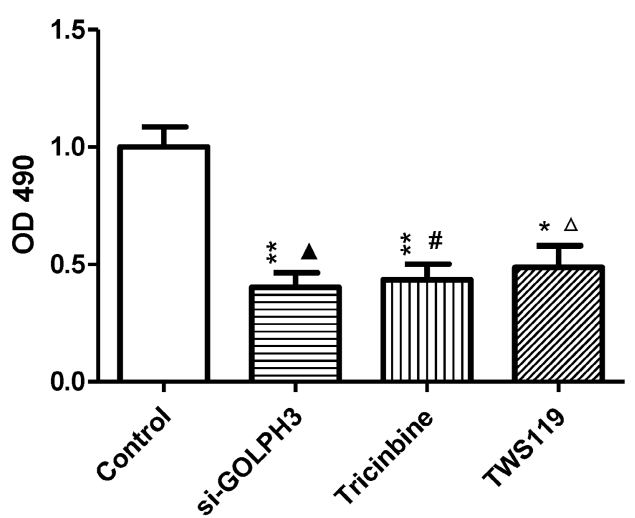

Figure 4. The influence of different processing on SW620 colon cancer cell proliferation inhibition. The cell growth inhibition rate was calculated by measuring the absorbance at the wavelength of $490 \mathrm{~nm}$. The growth inhibition rates of si-GOLPH3 group, Tricinbine group and TWS119 group were $59.9 \%$, $56.6 \%, 51.3 \%$, respectively. *: $P<0.05$, **: $P<0.01$ compared with control group; $\Delta: P>0.05$ compared with Tricinbine group; \#: $P>0.05$ compared with TWS119 group; $\triangle: P>0.05$ compared with si-Golph3 group.
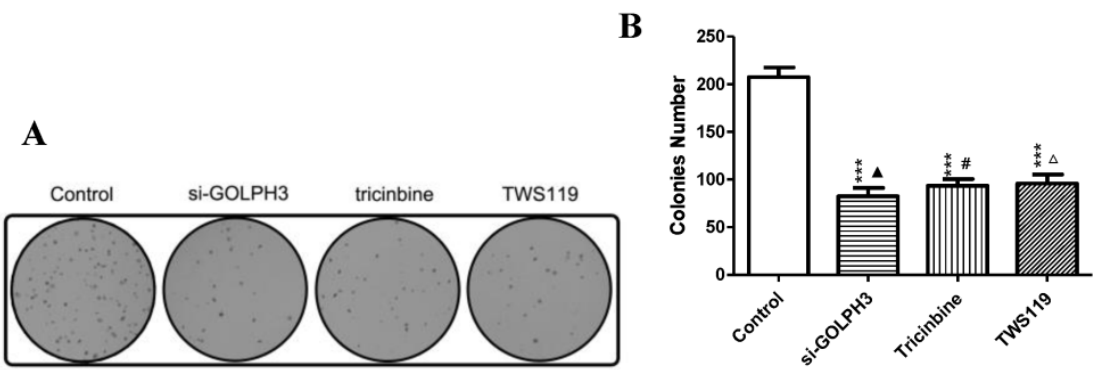

Figure 5. A. Colony image of SW620 colon cancer cells in each group. B. The comparison of SW620 colon cancer cell colony numbers. $* * *: P<0.001$ compared with control group; $\Delta$ : P>0.05 compared with Tricinbine group; \#: P>0.05 compared with TWS1 19 group; $\triangle$ : P>0.05 compared with si-Golph3 group. 
A

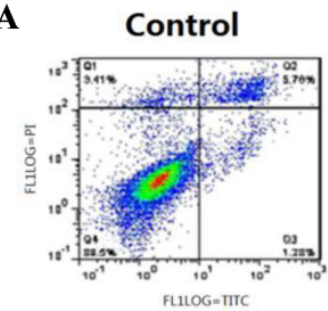

Tricinbine

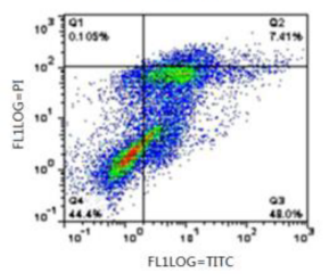

B

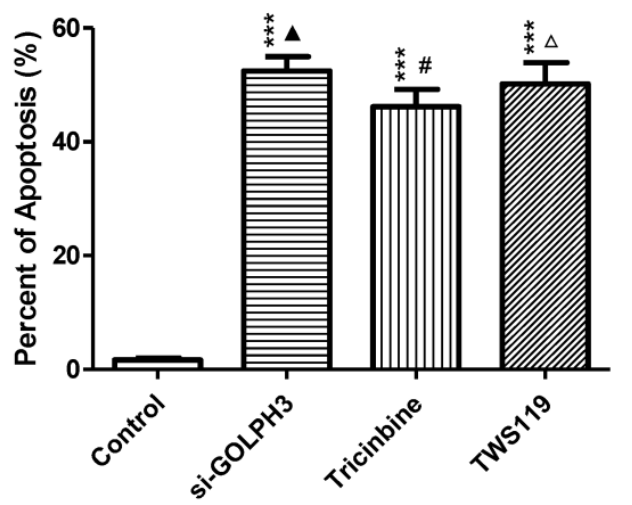

si-GOLPH3

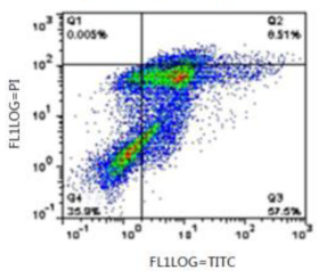

TWS119




signaling pathway of tumor cells, such as colorectal cancer cells, can be overactivated, which is closely related to the development of tumors ${ }^{11,12}$. As a new proto-oncogene, GOLPH3 can promote tumor cell proliferation by activating the $\mathrm{PI} 3 \mathrm{~K} / \mathrm{Akt} / \mathrm{mTOR}$ signaling pathway. However, research about the correlation between GOLPH3 and the Wnt/ $\beta$-catenin signaling pathway of colorectal cancer cells is still not being undertaken.

Our previous studies showed a positive correlation between excess expression of GOLPH3 protein in poorly differentiated human colorectal cancer cells, lymph node metastasis and advanced cancer stage. Besides, the expression of GOLPH3 protein was negatively correlated with apoptosis ${ }^{13-15}$. This phenomenon is associated with abnormal expression of $\beta$-catenin in colorectal cancer, which may activate the Wnt signaling pathway. Furthermore, we found that silencing expression of GOLPH3 gene inhibited the growth and proliferation of colon cancer cells and promoted apoptosis, through using transfection technology. Similar results have been reported for breast cancer, esophageal squamous cell carcinoma and other cancers ${ }^{16,17}$. At the same time, silencing GOLPH3 gene can decrease the activity of the Wnt signaling pathway and expression of $\beta$-catenin protein, which suggests that excess expression of GOLPH3 gene can active the Wnt signaling pathway and increase $\beta$-catenin protein expression, which promotes cancer cell proliferation.

Overexpression of GOLPH3 can activate Akt by phosphorylation of Ser473. Substrates of activated Akt include mTOR and GSK-3 $\beta^{18}$. As a regulatory factor of the Wnt/ $\beta$-catenin signaling pathway, GSK-3 $\beta$ is also involved in regulating cell proliferation and apoptosis by its effect on $\beta$-catenin ${ }^{19,20}$. We found that by silencing expression of GOLPH3 gene, blocking Akt and GSK3 $\beta$ can inhibit proliferation of colon cancer cells and activity of the Wnt signaling pathway, and promote apoptosis of SW620 cells. There was no significant difference in Wnt signaling pathway activity and proliferation and apoptosis of colon cancer cells in each experimental group. Therefore, we speculate that overexpression of GOLPH3 gene can promote the proliferation of colon cancer cells by activating the Wnt signaling pathway through Akt/GSK-3 $\beta / \beta$-catenin.

We found that expression of $\beta$-catenin in the group with added GSK-3 $\beta$ inhibitor was higher than that in the other two groups, but the expression of GSK-3 $\beta$ and pS9-GSK3 $\beta$ was not significantly different in each group. Therefore, we infer that GOLPH3 may also activate the Wnt signaling pathway by other means. Some studies have shown that GOLPH3 can affect the activity of the Wnt signaling pathway, mediated by VPS35, which is probably by regulating the transport of Wnt protein between endosomes and the Golgi ${ }^{4,21}$. Another possible mechanism may be by interfering with the recovery and recycling of Wntless, which plays a key role in Wnt protein secretion ${ }^{22,23}$. Therefore, further study of the relationship between GOLPH3 gene and Wnt signaling pathway in colorectal cancer cells is needed.

In conclusion, overexpression of GOLPH3 gene in SW620 colon cancer cells can promote cancer cell proliferation and inhibit apoptosis by activating the Wnt signaling pathway. The mechanism may be that overexpression of GOLPH3 gene activates Akt, and further activates the Wnt signaling pathway mediated by GSK-3 $\beta$. GOLPH3 can be used as a potential therapeutic target against colon cancer.

\section{Acknowledgements}

We wish to thank Prof. Huang Weifeng and members of the field laboratory for their helpful advice, reagents, and support. The study was supported by the Nursery Fund and Research Fund of the Second Affiliated Hospital of Fujian Medical University, and Fujian Natural Science Foundation of China.

\section{Competing Interests}

The authors declare that there is no competing interest related to this study.

\section{References}

1. Aronova S, Wedaman K, Anderson S, et al. Probing the membrane environment of the TOR kinases reveals functional interactions between TORC1, actin, and membrane trafficking in Saccharomyces cerevisiae. Mol Biol Cell. 2007;18:2779-2794.

2. Scott KL, Chin L. Signaling from the Golgi: mechanisms and models for Golgi phosphoprotein 3-mediated oncogenesis. Clin Cancer Res. 2010;16:2229-2234.

3. Farber-Katz SE, Dippold HC, Buschman MD, et al. DNA damage triggers Golgi dispersal via DNA-PK and GOLPH3. Cell. 2014;156:413-427.

4. Scott KL, Kabbarah O, Liang MC, et a1. GOLPH3 modulates mTOR signaling and rapamycin sensitivity in cancer. Nature. 2009;459:1085-1090.

5. Li WZ, Guo FF, Gu M, et a1. Increased Expression of GOLPH3 is Associated with the Proliferation of Prostate Cancer. J Cancer. 2015; 6: 420-429.

6. $\mathrm{Hu} \mathrm{BS}, \mathrm{Hu} \mathrm{H}$, Zhu CY, et al. Overexpression of Golph3 is associated with poor clinical outcome in gastric cancer. Tumour Biol J. 2013;34:515-520.

7. Peng JZ, Fang Y, Tao Y, et al. Mechanisms of GOLPH3 Associated with the Progression of Gastric Cancer: A Preliminary Study. PLoS One. 2014;9:e107362.

8. Dai T, Zhang D, Cai M, et al. Golgi phosphoprotein 3 (GOLPH3) promotes hepatocellular carcinoma cell aggressiveness by activating the NF-kB pathway. J Pathol. 2015; 235:490-501.

9. Hu GS, Li YQ, Yang YM, Shi W, Liao AJ, Yao YH, Zeng B, Yuan J. High expression of Golgi phosphoprotein-3 is associated with poor survival in patients with hepatocellular carcinoma. Tumour Biol. 2014;35:8625-8632.

10. Zhang LJ, Wang KB, Liu LS, et al. Overexpression of GOLPH3 is associated with poor prognosis and clinical progression in pancreatic ductal adenocarcinoma. BMC Cancer. 2014; 14: 571.

11. Nathália G. Amado, Danilo Predes, Marcela M. Moreno, et al. Flavonoids and Wnt/ $\beta$-Catenin Signaling: Potential Role in Colorectal Cancer Therapies. Int J Mol Sci. 2014; 15: 12094-12106.

12. A Syed Sameer, Zaffar A Shah, Safiya Abdullah, Nissar A Chowdri, Mushtaq A Siddiqi. Analysis of molecular aberrations of Wnt pathway gladiators in colorectal cancer in the Kashmiri population. Hum Genomics 2011; 5: 441-452.

13. Qiu CZ, Yu WS, Wang CX. Expression and clinical significance of Golgi phosphorylation protein 3 in colorectal cancer tissues. Chin J Exp Surg. 2013;30:461-463. 
14. Yu WS, Qiu CZ, Wang CX. Golph3 expression and apoptosis in colorectal cancer cells. J Clin Oncol. 2013;40:1094-1463.

15. Zhou ZP, Qiu CZ, Yu WS, Yang XF. Relationship between golgi phosphoprotein 3 expression and $\mathrm{Wnt} / \beta$-catenin signaling in colorectal cancer tissue. Chin J Exp Surg 2013; 30:2709-2710.

16. Wang JH, Chen XT, Wen ZS, et al. High Expression of GOLPH3 in Esophageal Squamous Cell Carcinoma Correlates with Poor Prognosis. PLoS One. 2012; 7: e45622.

17. Zeng $\mathrm{Z}$, Lin $\mathrm{H}, \mathrm{Zhao} \mathrm{X}$, et al. Overexpression of GOLPH3 promotes proliferation and tumorigenicity in breast cancer via suppression of the FOXO1 transcription factor. Clin Cancer Res. 2012;18:4059-4069.

18. Stefano Sechi, Anna Frappaolo, Giorgio Belloni, et al. The multiple cellular functions of the oncoprotein Golgi phosphoprotein 3. Oncotarget. 2015; 6: 3493-3506.

19. White BD, Chien AJ, Dawson DW. Dysregulation of Wnt/ $\beta$-catenin signaling in gastrointestinal cancers. Gastroenterology 2012;142:219-232.

20. Chen YG, Li Z, Wang XF. Where PI3K/Akt meets Smads:the crosstalk determines human embryonic stem cell fate. Cell Stem Cell. 2012;10:231-232.

21. Bugarcic A, Zhe Y, Kerr MC, Griffin J, Collins BM, Teasdale RD. Vps26A and Vps26B subunits define distinct retromer complexes. Traffic. 2011;12:1759-1773.

22. Belenkaya TY, Wu Y, Tang $\mathrm{X}$, et al. The retromer complex influences Wnt secretion by recycling wntless from endosomes to the trans-Golgi network. Dev Cell. 2008;14: 120-131.

23. Port F, Kuster M, Herr P, et al. Wingless secretion promotes and requires retromer-dependent cycling of Wntless. Nat Cell Biol. 2008;10: 178-185. 